\title{
Multiple cross-reactivity to several types of neuromuscular blocking agents in a patient with rocuronium anaphylaxis
}

\author{
Young-Jae Oh and Hyeon-Jeong Lee \\ Department of Anesthesia and Pain Medicine, School of Medicine, Pusan National University, Busan, Korea
}

Anaphylaxis that occurs during anesthesia is a major cause of morbidity and mortality. Muscle relaxant is the most frequent cause of perioperative anaphylaxis [1]. Although it is believed that there is a risk of anaphylaxis to the drug in some patients, a similar incidence of anaphylaxis has been observed with use of other members of the same drug class. We report on a case of anaphylactic reaction to rocuronium in a male patient confirmed as having multiple cross-reactivity to various neuromuscular blocking agents (NMBAs) on an allergic skin test at three months after discharge.

A 31-year-old, $174 \mathrm{~cm}, 85 \mathrm{~kg}$ man with congenital cystic adenomatoid malformation was scheduled to undergo lobectomy of the right lower lung. He did not have a history of allergy or surgery. Results of routine preoperative tests showed no abnormalities. In the operating room, routine monitoring devices, including a blood pressure (BP) cuff, pulse oximetry, and electrocardiography (ECG) were placed on the patient. He showed stable vital signs, including oxygen saturation $\left(\mathrm{SpO}_{2}\right) 100 \%$, BP $115 / 78 \mathrm{mmHg}$, and heart rate (HR) 90 beats/min. Epidural catheter insertion was performed for control of intraoperative and postoperative pain.

Propofol target-controlled infusion was performed for induction of general anesthesia. After loss of eyelash reflex, rocuronium $60 \mathrm{mg}$ was administered, and endotracheal intubation was performed after two minutes of facemask ventilation. After endotracheal intubation, his vital signs showed moderate hypotension; BP 85/40 mmHg, HR 75 beats/min. However, his breath sounds were normal. A 20-gauge arterial cannula was inserted into the left radial artery for continuous monitoring of arterial $\mathrm{BP}$ and a central venous catheter was placed in the right subclavian vein. He was treated with intravenous injection of ephedrine 10 $\mathrm{mg}$ and rapid infusion of Ringer's lactate solution. BP did not improve; therefore, we stopped propofol infusion. We injected midazolam $3 \mathrm{mg}$ and rocuronium $20 \mathrm{mg}$. A few minutes after injection, his vital signs indicated severe hypotension (BP 50/30 $\mathrm{mmHg}$ ). We observed a skin rash, urticaria, and edema on his face, neck, arms, and legs. On arterial blood gas analysis, the measured values were $\mathrm{pH}$ 7.230, $\mathrm{PCO}_{2} 50.4 \mathrm{mmHg}, \mathrm{PO}_{2} 124$ $\mathrm{mmHg}$ and $\mathrm{SpO}_{2}$ 98\%. The patient was treated with intravenous injection of epinephrine $0.1 \mathrm{mg}$ and continuous infusion of dopamine, dobutamine, and phenylephrine. After a few minutes of infusion, his vital signs became stable; BP 90/45 mmHg, HR 105 beats/min, and $\mathrm{SpO}_{2}$ 100\%. Hydrocortisone $100 \mathrm{mg}$ and chloropheniramine $4 \mathrm{mg}$ were administered intravenously. Seventy

Table 1. Results of Allergologic Test

\begin{tabular}{|c|c|c|c|c|c|}
\hline \multirow{2}{*}{ Drugs } & \multirow{2}{*}{ Prick test } & \multicolumn{4}{|c|}{ Intradermal test } \\
\hline & & $1: 1000$ & $1: 100$ & $1: 10$ & Undiluted \\
\hline Propofol & - & - & - & - & - \\
\hline Remifentanil & + & + & & & \\
\hline Lidocaine & - & - & - & - & - \\
\hline Rocuronium & + & - & + & & \\
\hline Vecuronium & + & - & + & & \\
\hline Ketamine & - & - & - & - & - \\
\hline Ropivacaine & - & - & - & - & - \\
\hline Atracurium & + & - & - & + & \\
\hline Fentanyl & - & - & - & - & - \\
\hline Succinylcholine & + & - & - & - & + \\
\hline Hextend & - & - & - & - & - \\
\hline Betadine & - & - & - & - & - \\
\hline
\end{tabular}

+ : positive test result, - : negative test result.

Corresponding author: Hyeon-Jeong Lee, M.D., Ph.D., Department of Anesthesia and Pain Medicine, School of Medicine, Pusan National University, 179, Gudeok-ro, Seo-gu, Busan 602-739, Korea. Tel: 82-51-240-7399, Fax: 82-51-242-7466, E-mail: 1hjksk@pusan.ac.kr (c) This is an open-access article distributed under the terms of the Creative Commons Attribution Non-Commercial License (http:// creativecommons.org/licenses/by-nc/3.0/), which permits unrestricted non-commercial use, distribution, and reproduction in any medium, provided the original work is properly cited. 
five minutes after induction of anesthesia, he was transferred to the intensive care unit. Five hours after the event, his vital signs became stable without inotropics and vasopressors. He was transferred to the general ward 13 hours after the event and discharged after three days without occurrence of other complications.

Allergic skin test for each drug was performed three months after discharge (Table 1). Skin prick and intradermal tests for remifentanil, rocuronium and vecuronium were positive. One month after the test, allergic skin test for other anesthetic agents was performed. The patient also showed a positive reaction on the skin test for atracurium and succinylcholine. We planned epidural anesthesia for planned surgery using negative skin tested drugs; however, the patient refused further treatment and was discharged.

IgE-mediated anaphylaxis by NMBAs and antibiotics commonly occurs during the perioperative period [2]. Potential allergic cross-reactivity among the drugs is frequently regarded as a risk factor for perioperative anaphylaxis [2]. Among the drugs, NMBAs are most frequently involved in anaphylaxis and crossreactivity commonly occurs between NMBAs [3].
Dong et al. [4] suggested that single sensitization was frequent in cases of allergy to succinylcholine, whereas it was less common with steroidal or benzylisoquinoline compounds. Crossreactivity studies appear to be of critical importance in cases of allergic reaction to an NMBA. However, despite a previously documented NMBA-induced anaphylaxis, negative skin tested NMBAs may be used safely [5]. As in this case, a patient showing multiple cross-reactivity to all types of NMBAs on allergic skin tests is a very rare example. In addition, other types of drugs, such as remifentanil, also exhibited reactivity. For these patients, use of negative intradermal tested drugs and use of regional anesthesia without muscle relaxant should be considered for future surgery.

As an anesthesiologist, prompt recognition and treatment of anaphylaxis is needed. In cases of suspected anaphylactic reaction, extensive investigation should be conducted through combined preoperative and postoperative tests in order to identify the nature of the reaction, and the responsibility of suspected drugs, and to provide recommendations for future administration of anesthesia.

\section{References}

1. Mertes PM, Laxenaire MC, Alla F. Anaphylactic and anaphylactoid reactions occurring during anesthesia in France in 1999-2000. Anesthesiology 2003; 99: 536-45.

2. Dewachter P, Mouton-Faivre C, Castells MC, Hepner DL. Anesthesia in the patient with multiple drug allergies: Are all allergies the same? Curr Opin Anaesthesiol 2011; 24: 320-5.

3. Dewachter P, Mouton-Faivre C, Emala CW. Anaphylaxis and anesthesia: controversies and new insights. Anesthesiology 2009; 111: 114150.

4. Dong SW, Mertes PM, Petitpain N, Hasdenteufel F, Malinovsky JM. Hypersensitivity reactions during anesthesia. Results from the ninth French survey (2005-2007). Minerva Anestesiol 2012; 78: 868-78.

5. Dewachter P, Mouton-Faivre C, Pertek JP, Boudaa C, Mertes PM. Value of skin tests for the choice of a neuromuscular blocking agent after an anaphylactic reaction. Ann Fr Anesth Reanim 2005; 24: 543-6. 